Available online at www.sciencedirect.com

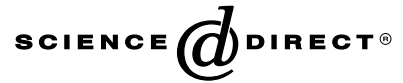

Multiphase Flow

ELSEVIER

International Journal of Multiphase Flow xxx (2005) xxx-xxx

www.elsevier.com/locate/ijmulflow

\title{
Ellipsoidal model of the rise of a Taylor bubble in a round tube
}

\author{
T. Funada a, D.D. Joseph ${ }^{\text {b,*, }}$ T. Maehara ${ }^{\text {a }}$, S. Yamashita ${ }^{\text {a }}$ \\ a Department of Digital Engineering, Numazu College of Technology, 3600 Ooka, Numazu, Shizuoka 410-8501, Japan \\ ${ }^{\mathrm{b}}$ Department of Aerospace Engineering and Mechanics, University of Minnesota, 110 Union St. SE, \\ Minneapolis, MN 55455, USA
}

Received 20 March 2004; received in revised form 29 November 2004

\begin{abstract}
The rise velocity of long gas bubbles (Taylor bubbles) in round tubes is modeled by an ovary ellipsoidal cap bubble rising in an irrotational flow of a viscous liquid. The analysis leads to an expression for the rise velocity which depends on the aspect ratio of the model ellipsoid and the Reynolds and Eötvös numbers. The aspect ratio of the best ellipsoid is selected to give the same rise velocity as the Taylor bubble at given values of the Eötvös and Reynolds numbers. The analysis leads to a prediction of the shape of the ovary ellipsoid which rises with same velocity as the Taylor bubble.
\end{abstract}

(C) 2005 Elsevier Ltd. All rights reserved.

Keywords: Taylor bubble; Ovary ellipsoidal model; Viscous potential flow

\section{Introduction}

The correlations given by Viana et al. (2003) convert all the published data on the normalized rise velocity $F r=U /(g D)^{1 / 2}$ into analytic expressions for the Froude velocity versus buoyancy Reynolds number, $R_{\mathrm{G}}=\left(D^{3} g\left(\rho_{\mathrm{L}}-\rho_{\mathrm{G}}\right) \rho_{\mathrm{L}}\right)^{1 / 2} / \mu$ for fixed ranges of the Eötvös number, $E_{o}=g \rho_{\mathrm{L}} D^{2} / \sigma$ where $D$ is the pipe diameter, $\rho_{\mathrm{L}}, \rho_{\mathrm{G}}$ and $\sigma$ are densities and surface tension. Their plots give rise to power laws in $E_{o}$; the composition of these separate power laws emerge as

\footnotetext{
${ }^{*}$ Corresponding author. Tel.: +1 612625 0309; fax: +1 6126261558.

E-mail address: joseph@aem.umn.edu (D.D. Joseph).
} 


\section{ARTICLE IN PRESS}

bi-power laws for two separate flow regions for large and small buoyancy Reynolds. For large $R_{\mathrm{G}}$ $(>200)$ they find that

$$
F r=0.34 /\left(1+3805 / E_{o}^{3.06}\right)^{0.58} .
$$

For small $R_{\mathrm{G}}(<10)$ they find

$$
F r=\frac{9.494 \times 10^{-3}}{\left(1+6197 / E_{o}^{2.561}\right)^{0.5793}} R_{\mathrm{G}}^{1.026} .
$$

The flat region for high buoyancy Reynolds number and sloped region for low buoyancy Reynolds number is separated by a transition region $\left(10<R_{\mathrm{G}}<200\right)$ which they describe by fitting the data to a logistic dose curve. Repeated application of logistic dose curves lead to a composition of rational fractions of power laws. This leads to the following universal correlation:

$$
F r=\frac{0.34 /\left(1+3805 / E_{o}^{3.06}\right)^{0.58}}{\left(1+\left(\frac{R_{\mathrm{G}}}{31.08}\left(1+\frac{778.76}{E_{o}^{1.96}}\right)^{-0.49}\right)^{-1.45\left(1+\frac{7.22 \times 10^{13}}{E_{o}^{9.93}}\right)^{0.094}}\right)^{0.71\left(1+\frac{7.22 \times 10^{13}}{E_{o}^{9.93}}\right)^{-0.094}} .}
$$

The performance of the universal correlation (1.3) is evaluated in Fig. 1 where the values predicted by (1.3) are compared to the experiments. Almost all of the values fall within the $20 \%$ error line and most of the data is within $10 \%$ of predicted values.

The formula (1.3) solves the problem of the rise velocity of Taylor bubbles in round pipes. This formula arises from processing data and not from flow fundamentals; one might say that the problem of the rise velocity has been solved without understanding.

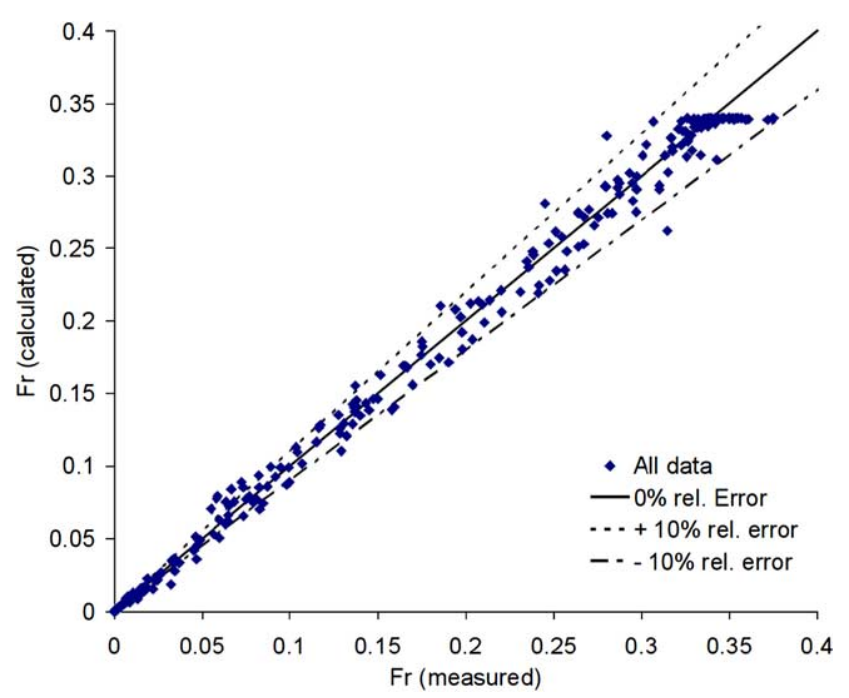

Fig. 1. Fr predicted from (1.3) vs. experimental data $\left(E_{o}>6\right)$. 


\section{ARTICLE IN PRESS}

\subsection{Unexplained and paradoxical features}

The teaching of fluid mechanics would lead one to believe that a bubble rising steadily in a liquid is in a balance of buoyant weight and drag. It is natural to think that the buoyant weight is proportional to the volume of gas, but the accurate formula (1.3) does not depend on the length of the bubble; this requires explanation.

Even the theoretical results are mysterious. The rise velocity $U$ of the spherical cap bubble at high Reynolds number is accurately determined from a potential flow analysis of motion in an inviscid fluid by Davies and Taylor (1950) and in a viscous fluid by Joseph (2003). Analysis of the rise velocity of Taylor bubbles in inviscid fluids based on shape of the bubble nose was given first by Dumitrescue (1943) and then by Davies and Taylor (1950).

Joseph (2003) found a formula for the rise velocity of a spherical cap bubble from analysis of interfacial balances at the nose of a bubble rising in irrotational flows of a viscous fluid. He found that

$$
\frac{U}{\sqrt{g D}}=-\frac{8}{3} \frac{v(1+8 s)}{\sqrt{g D^{3}}}+\frac{\sqrt{2}}{3}\left[1-2 s-\frac{16 s \sigma}{\rho g D^{2}}+\frac{32 v^{2}}{g D^{3}}(1+8 s)^{2}\right]^{1 / 2}
$$

where $R=D / 2$ is the radius of the cap, $\rho$ and $v$ are the density and kinematic viscosity of the liquid, $\sigma$ is surface tension, $r(\theta)=R\left(1+s \theta^{2}\right)$ and $s=r^{\prime \prime}(0) / D$ is the deviation of the free surface from perfect sphericity $r(\theta)=R$ near the stagnation point $\theta=0$. The bubble nose is more pointed when $s<0$ and blunted when $s>0$. A more pointed bubble increases the rise velocity; the blunter bubble rises slower.

The dependence of (1.4) on terms proportional to $s$ is incomplete because the potential solution for a sphere and the curvature for a sphere were not perturbed. A complete formula (2.39) replacing (1.4) is derived in Section 2.

The Davies and Taylor (1950) result arises when all other effects vanish; if $s$ alone is zero,

$$
\frac{U}{\sqrt{g D}}=-\frac{8}{3} \frac{v}{\sqrt{g D^{3}}}+\frac{\sqrt{2}}{3}\left[1+\frac{32 v^{2}}{g D^{3}}\right]^{1 / 2}
$$

showing that viscosity slows the rise velocity. Eq. (1.5) gives rise to a hyperbolic drag law

$$
C_{D}=6+32 / R
$$

which agrees with data on the rise of spherical cap bubbles given by Bhaga and Weber (1981).

It is unusual that the drag on the cap bubble plays no role in the analysis leading to (1.4). Batchelor (1967) notes that

...the remarkable feature of [equations like (1.4)] and its various extensions is that the speed of movement of the bubble is derived in terms of the bubble shape, without any need for consideration of the mechanism of the retarding force which balances the effect of the buoyancy force on a bubble in steady motion. That retarding force is evidently independent of Reynolds number, and the rate of dissipation of mechanical energy is independent of viscosity, implying that stresses due to turbulent transfer of momentum are controlling the flow pattern in the wake of the bubble. 


\section{ARTICLE IN PRESS}

This citation raises another anomalous feature about the rise of cap bubbles and Taylor bubbles relating to the wake. An examination of rise velocity from Bhaga and Weber (1981) and the study of Taylor bubbles in Viana et al. (2003) do not support the idea of turbulent transfer. The wake may be very turbulent as is true in water or apparently smooth and laminar as is true for bubbles rising in viscous oils but this feature does not enter into any of the formulas for the rise velocity, empirical as in (1.3) or theoretical as in (1.4).

A related paradoxical property is that the Taylor bubble rise velocity does not depend on how the gas is introduced into the pipe. In the Davies-Taylor experiments the bubble column is open to the gas. In other experiments the gas is injected into a column whose bottom is closed.

It can be said, despite successes, a good understanding of the fluid mechanics of the rise of cap bubbles and Taylor bubbles is not yet available.

\subsection{Drainage}

Many of the paradoxical features of the rise of Taylor bubbles can be explained by drainage in Fig. 2. The liquid at the wall drains under gravity with no pressure gradient. If the liquid is put into motion by a pressure gradient the gas bubble will deform and the film flow will not be governed by (1.7); the drain equation is

$$
\frac{\mu}{r} \frac{\mathrm{d}}{\mathrm{d} r}\left(r \frac{\mathrm{d} u}{\mathrm{~d} r}\right)=\rho_{\mathrm{L}} g
$$

subject to no slip at the wall and no shear at the bubble surface.

It can be argued that the cylindrical part of the long bubble is effectively not displacing liquid since the pressure does not vary along the cylinder. In this case buoyant volume entering into the equation buoyancy = drag would be the vaguely defined hemisphere poking into the liquid at top. The source of drag is unclear; since shear stresses do not enter the drag ought to be determined by the vertical projections of normal stresses all around the bubble. This kind of analysis has not appeared in the literature. A different kind of analysis, depending on the shape of the bubble and sidewall drainage has been applied. This kind of analysis leads ultimately to a formula for the rise velocity of the bubble nose. Apparently the shape of the bubble nose is an index of the underlying drag balance.

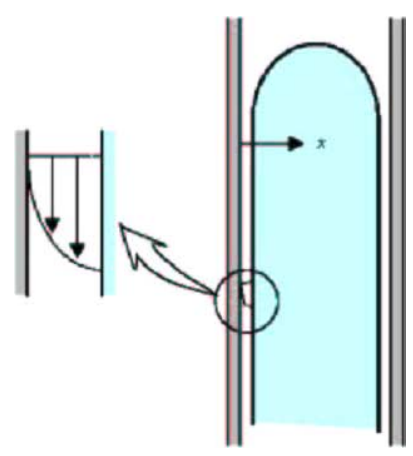

Fig. 2. Drainage at the wall of a rising Taylor bubble. If $U$ is added to this system the wall moves and the bubble is stationary. 


\section{ARTICLE IN PRESS}

\subsection{Brown's 1965 analysis of drainage}

Brown (1965) put forward a model of the rise velocity of large gas bubbles in tubes. A similar model was given by Batchelor (1967). There are two elements for this model.

(1) The rise velocity is assumed to be given by $C \sqrt{g R_{\delta}}$ where $C(=0.494)$ is an empirical constant and $R_{\delta}=R-\delta$ is the bubble radius, $R$ is the tube radius and $\delta$ is the unknown film thickness.

(2) It is assumed that the fluid drains in a falling film of constant thickness $\delta$. The film thickness is determined by conserving mass: the liquid displaced by the rising bubble must balance the liquid draining at the wall.

After equating two different expressions for the rise velocity arising from (1) and (2), Brown finds that

$$
U=0.35 V \sqrt{1-\frac{2(\sqrt{1+\psi}-1)}{\psi}},
$$

where

$$
\psi=\left(14.5 \Re^{2}\right)^{1 / 3}, \quad \mathfrak{R}=V D / v, \quad V=\sqrt{g D} .
$$

The expression for the rise velocity (1.8) does not account for effects of surface tension which are negligible when the bubble radius is large. The expression is in moderately good agreement with data, but not nearly as good as the correlation formula (1.3).

The rise velocity $\sqrt{g R_{\delta}}$ is still determined by the bubble shape, but that shape is altered by drainage.

\subsection{Viscous Potential flow}

We have already mentioned that the correlation formula (1.3) accurately predicts the rise velocity and further improvement cannot be expected from modeling. Our understanding of the fluid mechanics under way is however far from complete. When surface tension is neglected the formula (1.5) extends the results of Dumitrescue (1943) and Davies and Taylor (1950) from inviscid fluids to viscous fluids by assuming that the cap of the bubble remains spherical, even at finite Reynolds number. The same extension to include the effects of viscosity in the formula for the rise velocity based on potential flow at the nose should be possible for Taylor bubbles if the nose remains spherical in viscous fluids. Brown says that Fig. 3 (note that he used $R_{c}$ for $R-\delta$, which is $R_{\delta}$ in our nomenclature.)

"... indicates that although the cavity shapes are different in the transition region, they are remarkably similar in the nose region. The second interesting fact... is that the frontal radius of the cavity in normalized coordinates $\left(R_{c}=R-\delta\right)$ which is the same for all liquids, is 0.75 , the same value as was obtained in the analysis of bubbles in inviscid liquids. 


\section{ARTICLE IN PRESS}

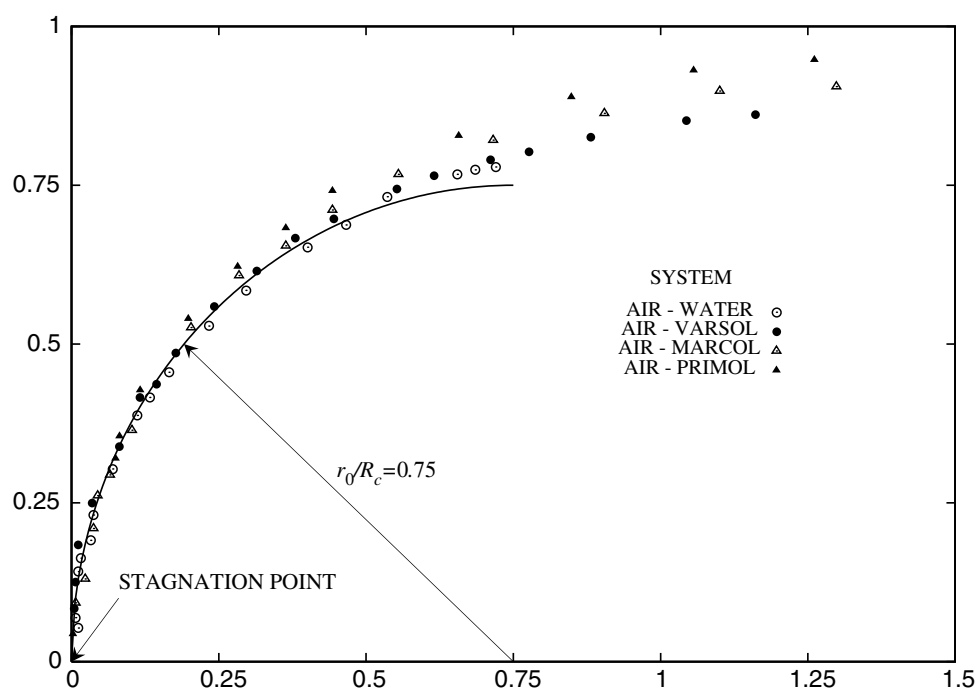

Fig. 3. (After Brown, 1965). The profile of the cap of Taylor bubbles. The nose region is spherical with a radius $r_{0}$. For all the fluids, $r_{0} / R_{c}=0.75$. The viscosities of water, varsol, marcol, and primol apparently are $0.977,0.942,19.42$ and $142.3 \mathrm{mPa}$ s respectively.

The scatter in the data plotted in Fig. 3 and the data in the transition can be fit even better by the cap of an ovary ellipsoid which is nearly spherical (Fig. 10) arising from the analysis given in Section 2.

The effect of surface tension is to retard the rise velocity of Taylor bubbles in round tubes. The universal correlation (1.3) shows that the rise velocity decreases as the Eötvös number $E_{o}=g \rho_{\mathrm{L}} D^{2} / \sigma$ decreases, for ever smaller values of $D^{2} / \sigma$. In fact these kinds of bubbles, with large tensions in small tubes, do not rise; they stick in the pipe preventing draining. If the radius of a stagnant bubble $R=2 \sigma / \Delta p$ with the same pressure difference $\Delta p$ as in the Taylor bubble, is larger than the tube radius, it will plug the pipe. White and Beardmore (1962) said that the bubble will not rise when $E_{o}<4$. This can be compared with the values 3.36 given by Hattori (1935), 3.37 given by Bretherton (1961), 5.8 given by Barr (1926), and 4 given by Gibson (1913).

A very convincing set of experiments showing the effect of drainage is reported for "Taylor bubbles in miniaturized circular and noncircular channels" by Bi and Zhao (2001). They showed that for triangular and rectangular channels, elongated bubbles always rose upward even though the hydraulic diameter of the tube was as small as $0.866 \mathrm{~mm}$, whereas in circular tubes the bubble motion stopped when $d \leqslant 2.9 \mathrm{~mm}$. They did not offer an explanation but the reason is that surface tension cannot close the sharp corners where drainage can occur.

\section{Ellipsoidal bubbles}

Grace and Harrison (1967) studied rise of ellipsoidal bubble in an inviscid liquid. They sought to explain the influence of bubble shape on the rise velocity and concluded that elliptical cap and ovary ellipsoidal bubbles rise faster than the corresponding circular-cap and spherical-cap bub- 
bles. They cited experimental data which they claim support their results. They say that “ $\ldots$ bubbles take up elliptical shapes if they enclose a surface (e.g. a rod)." This statement is not correct because bubbles rising in the presence of a central rod are usually not axisymmetric as can be seen in Fig. 4.

In this paper we shall obtain expressions for the rise velocity of ovary and planetary ellipsoids. The ovary ellipsoid looks more like a long bubble than a planetary ellipsoid (Fig. 5). There is no way that the planetary ellipsoid can be fit to the data given by Viana et al. (2003), but the ovary ellipsoid can be made to fit Viana's data with one shape parameter for all cases. This is very surprising, since the Taylor bubble is not thought to be ellipsoidal and the dynamics of these bubbles is controlled by sidewall drainage which is entirely neglected in the following potential flow analysis of the rise of ellipsoidal bubbles in a viscous liquid.

Ovary and planetary ellipsoidal bubbles are shown in Fig. 5. We will be led by the analysis to cases in which the ovary ellipsoids are nearly spherical with $D=2 a$.

For axisymmetric flows of incompressible fluid around the ellipsoid of revolution, we can have the stream function and the velocity potential, then we have the solution which satisfies the kinematic condition at the surface of the bubble and the normal stress balance there which contains the viscous normal stress based on viscous potential flow.

\subsection{Ovary ellipsoid}

In an ellipsoidal frame $(\xi, \eta, \varphi)$ on the ovary ellipsoid bubble moving with a uniform velocity $U$ in a liquid, we have the stream function $\psi$ and the velocity potential $\phi$ for axisymmetric flow.

$$
\psi=\frac{1}{2} U c^{2} \sin ^{2} \eta\left[\sinh ^{2} \xi-\frac{b^{2}}{a^{2} K}\left(\cosh \xi+\sinh ^{2} \xi \ln \tanh \frac{\xi}{2}\right)\right]
$$

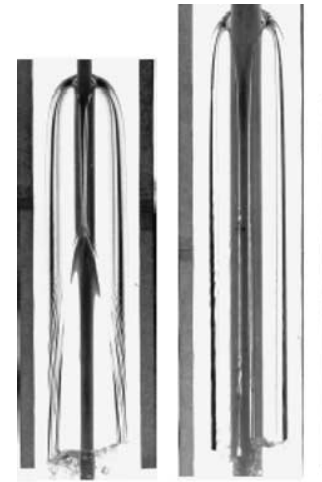

(a) (b)

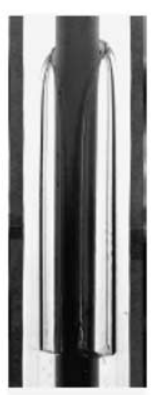

(c)

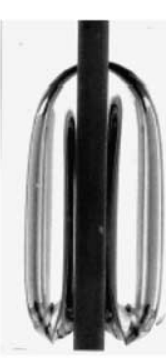

(d)

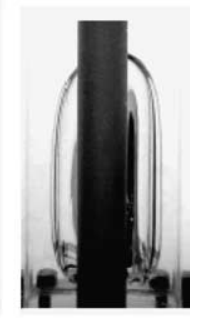

(e)

Fig. 4. Photographs (unpublished, courtesy of F. Viana and R. Pardo) of Taylor bubbles rising in concentric annular space of $76.2 \mathrm{~mm}$ inside diameter pipe and different rod diameter (ID) filled with different viscous liquids: (a) water $\left(1 \mathrm{mPa} \mathrm{s}, 997 \mathrm{~kg} / \mathrm{m}^{3}\right), \mathrm{ID}=12.7 \mathrm{~mm}$; (b) water, ID = $25.4 \mathrm{~mm}$; (c) water, ID = $38.1 \mathrm{~mm}$; (d) silicone oil (1300 mPa s, $\left.970 \mathrm{~kg} / \mathrm{m}^{3}\right), \mathrm{ID}=12.7 \mathrm{~mm}$; (e) silicone oil $\left(1300 \mathrm{mPa} \mathrm{s}, 970 \mathrm{~kg} / \mathrm{m}^{3}\right)$, ID $=25.4 \mathrm{~mm}$. The gas bubbles do not wrap all the way around the inner cylinder; a channel is opened for liquid drainage. 


\section{ARTICLE IN PRESS}
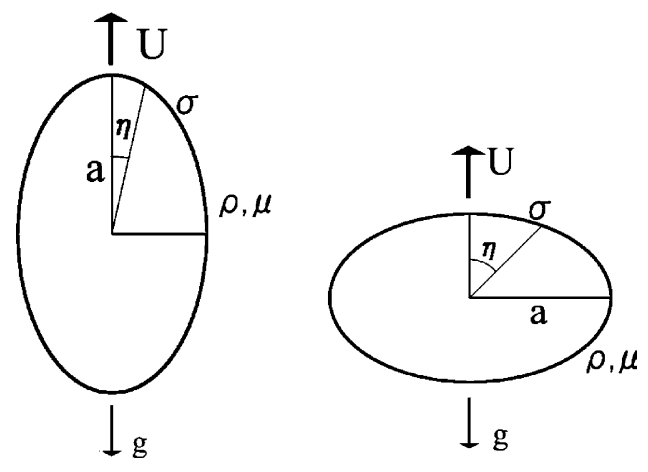

Fig. 5. An ellipsoid bubble moving with a uniform velocity $U$ in the $z$-direction of Cartesian coordinates $(x, y, z)$. An ovary ellipsoid is depicted in the left-hand side and a planetary ellipsoid is in the right-hand side, which are of the major semiaxis $a$, the minor semiaxis $b$, the aspect ratio $e=c / a, c^{2}=a^{2}-b^{2}$, and in a liquid (water) of density $\rho$, viscosity $\mu$, with the surface tension $\sigma$ at the surface given by $\xi=\xi_{0}$ and under the acceleration due to gravity $g$.

$$
\phi=U c \cos \eta\left[\cosh \xi-\frac{b^{2}}{a^{2} K}\left(1+\cosh \xi \ln \tanh \frac{\xi}{2}\right)\right],
$$

with $c^{2}=a^{2}-b^{2}, e=c / a$ and $K$ :

$$
K=e^{2}\left[\frac{a}{c}+\frac{b^{2}}{c^{2}} \ln \frac{a+b-c}{a+b+c}\right]=\frac{1}{\cosh \xi_{0}}+\tanh ^{2} \xi_{0} \ln \tanh \xi_{0} / 2=e+\left(1-e^{2}\right) \tanh ^{-1}(e) .
$$

(In this case, we take $z=c \cosh \xi \cos \eta, \varpi=c \sinh \xi \sin \eta, x=\varpi \cos \varphi$ and $y=\varpi \sin \varphi ; a=c \cosh \xi_{0}$ and $b=c \sinh \xi_{0}$.) The stream function (2.1) has been derived based on the article in Section 16.57 of Milne-Thomson's book (Milne-Thomson, 1996). The velocity $\boldsymbol{u}=\left(u_{\xi}, u_{\eta}\right)$ is expressed as

$$
\begin{aligned}
& u_{\xi}=-\frac{1}{J} \frac{\partial \phi}{\partial \xi}=-\frac{1}{\varpi J} \frac{\partial \psi}{\partial \eta}=-\frac{U c}{J} \cos \eta\left[\sinh \xi-\frac{b^{2}}{a^{2} K}(\operatorname{coth} \xi+\sinh \xi \ln \tanh \xi / 2)\right], \\
& u_{\eta}=-\frac{1}{J} \frac{\partial \phi}{\partial \eta}=\frac{1}{J \varpi} \frac{\partial \psi}{\partial \xi}=\frac{U c}{J} \sin \eta\left[\cosh \xi-\frac{b^{2}}{a^{2} K}(1+\cosh \xi \ln \tanh \xi / 2)\right] \equiv \frac{U c \sin \eta}{J} f_{1}(\xi), \\
& \frac{1}{J} \frac{\partial u_{\xi}}{\partial \xi}=-\frac{1}{J^{2}} \frac{\partial^{2} \phi}{\partial \xi^{2}}+\frac{1}{J^{3}} \frac{\partial J}{\partial \xi} \frac{\partial \phi}{\partial \xi} \\
& =-\frac{U c}{J^{2}} \cos \eta\left[\cosh \xi-\frac{b^{2}}{a^{2} K}\left(1-\frac{1}{\sinh ^{2} \xi}+\cosh \xi \ln \tanh \xi / 2\right)\right]-\frac{u_{\xi}}{J^{2}} \frac{\partial J}{\partial \xi} \\
& =-\frac{U c \cos \eta}{J^{2}} f_{2}(\xi)-\frac{u_{\xi}}{J^{2}} \frac{\partial J}{\partial \xi},
\end{aligned}
$$




\section{ARTICLE IN PRESS}

$$
\begin{aligned}
\left(\frac{1}{J} \frac{\partial u_{\xi}}{\partial \xi}\right)_{\xi_{0}} & =-U c \cos \eta\left[\frac{1}{J^{2}}\left[\cosh \xi-\frac{b^{2}}{a^{2} K}\left(1-\frac{1}{\sinh ^{2} \xi}+\cosh \xi \ln \tanh \xi / 2\right)\right]\right]_{\xi_{0}} \\
& \equiv-\frac{U c \cos \eta}{J_{0}^{2}} f_{2}\left(\xi_{0}\right),
\end{aligned}
$$

with

$$
\left.\begin{array}{l}
J^{2}=c^{2} \sinh ^{2} \xi+c^{2} \sin ^{2} \eta, \quad J_{0}=(J)_{\xi_{0}}, \\
f_{1}\left(\xi_{0}\right)=\cosh \xi_{0}-\frac{b^{2}}{a^{2} K}\left(1+\cosh \xi_{0} \ln \tanh \xi_{0} / 2\right)=\frac{e^{2}}{e+\left(1-e^{2}\right) \tanh ^{-1}(e)}=f_{1}(e), \\
f_{2}\left(\xi_{0}\right)=\cosh \xi_{0}-\frac{b^{2}}{a^{2} K}\left(1-\frac{1}{\sinh ^{2} \xi_{0}}+\cosh \xi_{0} \ln \tanh \xi_{0} / 2\right)=f_{2}(e)=2 f_{1}(e) .
\end{array}\right\}
$$

Following Joseph (2003), Bernoulli function is given by his (1.2) and (1.3):

$$
\frac{\rho|\boldsymbol{u}|^{2}}{2}+\Gamma=\frac{\rho U^{2}}{2}, \quad \frac{\rho_{\mathrm{G}}|\boldsymbol{u}|^{2}}{2}+\Gamma_{\mathrm{G}}=C_{\mathrm{G}}
$$

Put $\rho_{\mathrm{G}}|\boldsymbol{u}|^{2}=0$ in the gas. Then $\Gamma_{\mathrm{G}}=C_{\mathrm{G}}$ is constant. Boundary conditions at the surface of the ellipsoid (where $\xi=\xi_{0}$ ) are the kinematic condition and the normal stress balance

$$
u_{\xi}=0, \quad-[[\Gamma]]-[[\rho]] g h+[[2 \mu \boldsymbol{n} \cdot \mathbf{D}[\boldsymbol{u}]]] \cdot \boldsymbol{n}=-\sigma \nabla \cdot \boldsymbol{n},
$$

where $\Gamma=p+\rho g h$ as in Joseph (2003), the normal viscous stress $2 \mu \boldsymbol{n} \cdot \mathbf{D}[\boldsymbol{u}] \cdot \boldsymbol{n}$ and the normal vector $\boldsymbol{n}$ satisfy the following relations:

$$
\begin{aligned}
& {[[\Gamma]]=\Gamma_{\mathrm{G}}-\Gamma=C_{\mathrm{G}}-\frac{\rho}{2} U^{2}+\frac{\rho}{2}|\boldsymbol{u}|^{2}=C_{\mathrm{G}}-\frac{\rho}{2} U^{2}+\frac{\rho}{2}\left(u_{\eta}\right)_{\xi_{0}}^{2},} \\
& {[[2 \mu \boldsymbol{n} \cdot \mathbf{D}[\boldsymbol{u}]]] \cdot \boldsymbol{n}=-2 \mu\left(\frac{1}{J} \frac{\partial u_{\xi}}{\partial \xi}+u_{\eta} \frac{c^{2}}{J^{3}} \cos \eta \sin \eta\right)_{\xi_{0}},} \\
& \nabla \cdot \boldsymbol{n}=\frac{\operatorname{coth} \xi_{0}}{J_{0}^{3}}\left(J_{0}^{2}+c^{2} \sinh ^{2} \xi_{0}\right) .
\end{aligned}
$$

The normal stress balance is then expressed as

$$
-C_{\mathrm{G}}+\frac{\rho}{2} U^{2}-\frac{\rho}{2}\left(u_{\eta}\right)_{\xi_{0}}^{2}+\rho g h-2 \mu\left(\frac{1}{J} \frac{\partial u_{\xi}}{\partial \xi}+u_{\eta} \frac{c^{2}}{J^{3}} \cos \eta \sin \eta\right)_{\xi_{0}}=-\sigma \nabla \cdot \boldsymbol{n},
$$

where the distance from the top of the ellipsoid bubble is

$$
h=a(1-\cos \eta)=c \cosh \xi_{0}(1-\cos \eta) .
$$


For small $\eta$, we have

$$
\begin{aligned}
& J_{0}=c \sinh \xi_{0}\left(1+\frac{\eta^{2}}{2 \sinh ^{2} \xi_{0}}+O\left(\eta^{4}\right)\right), \\
& h=c \cosh \xi_{0}\left[\frac{1}{2} \eta^{2}+O\left(\eta^{4}\right)\right] \\
& \left(u_{\eta}\right)_{\xi_{0}}=\frac{U f_{1}\left(\xi_{0}\right)}{\sinh \xi_{0}}\left[\eta+O\left(\eta^{3}\right)\right] \\
& \left(\frac{1}{J} \frac{\partial u_{\xi}}{\partial \xi}\right)_{\xi_{0}}=-\frac{U f_{2}\left(\xi_{0}\right)}{c \sinh ^{2} \xi_{0}}\left[1-\left(\frac{1}{2}+\frac{1}{\sinh ^{2} \xi_{0}}\right) \eta^{2}+O\left(\eta^{4}\right)\right], \\
& \nabla \cdot \boldsymbol{n}=\frac{2 \cosh \xi_{0}}{c \sinh ^{2} \xi_{0}}\left(1-\frac{\eta^{2}}{\sinh ^{2} \xi_{0}}+O\left(\eta^{4}\right)\right) .
\end{aligned}
$$

Substitution of (2.16) into the normal stress balance (2.14) leads to a formula for the ovary bubble:

$$
\begin{aligned}
& -C_{\mathrm{G}}+\frac{\rho}{2} U^{2}-\frac{\rho}{2}\left(u_{\eta}\right)_{\xi_{0}}^{2}+\rho g h-2 \mu\left(\frac{1}{J} \frac{\partial u_{\xi}}{\partial \xi}+u_{\eta} \frac{c^{2}}{J^{3}} \cos \eta \sin \eta\right)_{\xi_{0}} \\
& =-\sigma \frac{2 \cosh \xi_{0}}{\operatorname{coshh}^{2} \xi_{0}}\left(1-\frac{\eta^{2}}{\sinh ^{2} \xi_{0}}\right) .
\end{aligned}
$$

Thus, we have $C_{\mathrm{G}}$ in $O(1)$

$$
C_{\mathrm{G}}=\frac{\rho}{2} U^{2}+\frac{2 \mu U f_{2}\left(\xi_{0}\right)}{c \sinh ^{2} \xi_{0}}+\sigma \frac{2 \cosh \xi_{0}}{c \sinh ^{2} \xi_{0}}
$$

and the following relation in $O\left(\eta^{2}\right)$

$$
-\frac{\rho}{2} \frac{U^{2} f_{1}^{2}\left(\xi_{0}\right)}{\sinh ^{2} \xi_{0}}+\frac{\rho g c}{2} \cosh \xi_{0}-\frac{2 \mu U}{c \sinh ^{2} \xi_{0}}\left[f_{2}\left(\xi_{0}\right)\left(\frac{1}{2}+\frac{1}{\sinh ^{2} \xi_{0}}\right)+\frac{f_{1}\left(\xi_{0}\right)}{\sinh ^{2} \xi_{0}}\right]=\sigma \frac{2 \cosh \xi_{0}}{\operatorname{csinh}^{4} \xi_{0}} .
$$

\subsection{Planetary ellipsoid}

In an ellipsoidal frame $(\xi, \eta, \varphi)$ on the planetary ellipsoid bubble moving with a uniform velocity $U$ in a liquid, we have the stream function $\psi$ and the velocity potential $\phi$ for axisymmetric flows

$$
\begin{aligned}
& \psi=\frac{1}{2} U c^{2} \sin ^{2} \eta\left[\cosh ^{2} \xi-\frac{\sinh \xi-\cosh ^{2} \xi \cot ^{-1} \sinh \xi}{e \sqrt{1-e^{2}}-\sin ^{-1} e}\right], \\
& \phi=U c \cos \eta\left[\sinh \xi-\frac{1-\sinh \xi \cot ^{-1} \sinh \xi}{e \sqrt{1-e^{2}}-\sin ^{-1} e}\right],
\end{aligned}
$$

where $c^{2}=a^{2}-b^{2}$ and $e=c / a$. (In this case, we take $z=c \sinh \xi \cos \eta, \varpi=c \cosh \xi \sin \eta$, $x=\varpi \cos \varphi$ and $y=\varpi \sin \varphi ; a=c \cosh \xi_{0}$ and $b=c \sinh \xi_{0}$.) The velocity $\boldsymbol{u}=\left(u_{\xi}, u_{\eta}\right)$ is given by

$$
u_{\xi}=-\frac{1}{J} \frac{\partial \phi}{\partial \xi}=-\frac{U c}{J} \cos \eta\left[\cosh \xi-\frac{\tanh \xi-\cosh \xi \cot ^{-1} \sinh \xi}{e \sqrt{1-e^{2}}-\sin ^{-1} e}\right]
$$




\section{ARTICLE IN PRESS}

$$
\begin{aligned}
u_{\eta}=-\frac{1}{J} \frac{\partial \phi}{\partial \eta}=\frac{U c}{J} \sin \eta\left[\sinh \xi-\frac{1-\sinh \xi \cot ^{-1} \sinh \xi}{e \sqrt{1-e^{2}}-\sin ^{-1} e}\right] \equiv \frac{U c \sin \eta}{J} f_{1}(\xi) \\
\frac{1}{J} \frac{\partial u_{\xi}}{\partial \xi}=-\frac{1}{J^{2}} \frac{\partial^{2} \phi}{\partial \xi^{2}}+\frac{1}{J^{3}} \frac{\partial J}{\partial \xi} \frac{\partial \phi}{\partial \xi} \\
=-\frac{U c}{J^{2}} \cos \eta\left[\sinh \xi-\frac{1+1 / \cosh ^{2} \xi-\sinh \xi \cot ^{-1} \sinh \xi}{e \sqrt{1-e^{2}}-\sin ^{-1} e}\right]-\frac{u_{\xi}}{J^{2}} \frac{\partial J}{\partial \xi} \\
=-\frac{U c \cos \eta}{J^{2}} f_{2}(\xi)-\frac{u_{\xi}}{J^{2}} \frac{\partial J}{\partial \xi},
\end{aligned}
$$$$
\left(\frac{1}{J} \frac{\partial u_{\xi}}{\partial \xi}\right)_{\xi_{0}}=-U c \cos \eta\left[\frac{1}{J^{2}}\left[\sinh \xi-\frac{1+1 / \cosh ^{2} \xi-\sinh \xi \cot ^{-1} \sinh \xi}{e \sqrt{1-e^{2}}-\sin ^{-1} e}\right]\right]_{\xi_{0}}
$$$$
\equiv-\frac{U c \cos \eta}{J_{0}^{2}} f_{2}\left(\xi_{0}\right)
$$

with

$$
\left.\begin{array}{l}
J^{2}=c^{2} \sinh ^{2} \xi+c^{2} \cos ^{2} \eta, \quad J_{0}=(J)_{\xi_{0}}, \\
f_{1}\left(\xi_{0}\right)=\sinh \xi_{0}-\frac{1-\sinh \xi_{0} \cot ^{-1} \sinh \xi_{0}}{e \sqrt{1-e^{2}}-\sin ^{-1} e}=\frac{-e^{2}}{e \sqrt{1-e^{2}}-\sin ^{-1}(e)}=f_{1}(e), \\
f_{2}\left(\xi_{0}\right)=\sinh \xi_{0}-\frac{1+1 / \cosh ^{2} \xi_{0}-\sinh \xi_{0} \cot ^{-1} \sinh \xi_{0}}{e \sqrt{1-e^{2}}-\sin ^{-1} e}=f_{2}(e)=2 f_{1}(e) .
\end{array}\right\}
$$

Boundary conditions at the surface of the ellipsoid (where $\xi=\xi_{0}$ ) are the kinematic condition and the normal stress balance

$$
\left.\begin{array}{l}
u_{\xi}=0 \\
-C_{\mathrm{G}}+\frac{\rho}{2} U^{2}-\frac{\rho}{2} u_{\eta}^{2}+\rho g h-2 \mu\left(\frac{1}{J} \frac{\partial u_{\xi}}{\partial \xi}-u_{\eta} \frac{c^{2}}{J^{3}} \cos \eta \sin \eta\right)=-\sigma \nabla \cdot \boldsymbol{n},
\end{array}\right\}
$$

where the distance from the top of the ellipsoid bubble and $\nabla \cdot \boldsymbol{n}$ are given, respectively, by

$$
h=b(1-\cos \eta)=c \sinh \xi_{0}(1-\cos \eta), \quad \nabla \cdot \boldsymbol{n}=\frac{\tanh \xi_{0}}{J_{0}^{3}}\left(J_{0}^{2}+c^{2} \cosh ^{2} \xi_{0}\right) .
$$


For small $\eta$, we have

$$
\begin{aligned}
& J_{0}=c \cosh \xi_{0}\left(1-\frac{\eta^{2}}{2 \cosh ^{2} \xi_{0}}+O\left(\eta^{4}\right)\right), \\
& h=c \sinh \xi_{0}\left[\frac{1}{2} \eta^{2}+O\left(\eta^{4}\right)\right], \\
& \left(u_{\eta}\right)_{\xi_{0}}=\frac{U f_{1}\left(\xi_{0}\right)}{\cosh \xi_{0}}\left[\eta+O\left(\eta^{3}\right)\right], \\
& \left(\frac{1}{J} \frac{\partial u_{\xi}}{\partial \xi}\right)_{\xi_{0}}=-\frac{U f_{2}\left(\xi_{0}\right)}{c \cosh ^{2} \xi_{0}}\left[1-\left(\frac{1}{2}-\frac{1}{\cosh ^{2} \xi_{0}}\right) \eta^{2}+O\left(\eta^{4}\right)\right], \\
& \nabla \cdot \boldsymbol{n}=\frac{2 \sinh ^{\xi_{0}} \cosh ^{2} \xi_{0}}{\cos _{0}}\left(1+\frac{\eta^{2}}{\cosh ^{2} \xi_{0}}+O\left(\eta^{4}\right)\right) .
\end{aligned}
$$

Substitution of these into the normal stress balance leads to a formula for the planetary bubble:

$$
\begin{aligned}
& -C_{\mathrm{G}}+\frac{\rho}{2} U^{2}-\frac{\rho}{2}\left(u_{\eta}\right)_{\xi_{0}}^{2}+\rho g h-2 \mu\left(\frac{1}{J} \frac{\partial u_{\xi}}{\partial \xi}-u_{\eta} \frac{c^{2}}{J^{3}} \cos \eta \sin \eta\right)_{\xi_{0}} \\
& =-\sigma \frac{2 \sinh \xi_{0}}{\cosh ^{2} \xi_{0}}\left(1+\frac{\eta^{2}}{\cosh ^{2} \xi_{0}}\right) .
\end{aligned}
$$

Thus we have $C_{\mathrm{G}}$ in $O(1)$

$$
C_{\mathrm{G}}=\frac{\rho}{2} U^{2}+\frac{2 \mu U f_{2}\left(\xi_{0}\right)}{c \cosh ^{2} \xi_{0}}+\sigma \frac{2 \sinh \xi_{0}}{\cosh ^{2} \xi_{0}}
$$

and the following relation in $O\left(\eta^{2}\right)$

$$
-\frac{\rho}{2} \frac{U^{2} f_{1}^{2}\left(\xi_{0}\right)}{\cosh ^{2} \xi_{0}}+\frac{\rho g c}{2} \sinh \xi_{0}-\frac{2 \mu U}{c \cosh ^{2} \xi_{0}}\left[f_{2}\left(\xi_{0}\right)\left(\frac{1}{2}-\frac{1}{\cosh ^{2} \xi_{0}}\right)-\frac{f_{1}\left(\xi_{0}\right)}{\cosh ^{2} \xi_{0}}\right]=-\sigma \frac{2 \sinh \xi_{0}}{c \cosh ^{4} \xi_{0}} .
$$

\subsection{Dimensionless rise velocity}

By taking the major axis $D=2 a$ as a representative length scale, $\sqrt{g D}$ as a velocity scale, $D / \sqrt{g D}$ as a time scale, the parameters involved in the expanded solution of dimensionless form are

Froude number: $F r=\frac{U}{\sqrt{g D}}$, Gravity Reynolds number: $R_{\mathrm{G}}=\frac{\sqrt{g D^{3}}}{v}$,

Eötvös number: $E_{o}=\frac{\rho g D^{2}}{\sigma}$, aspect ratio: $e=\frac{c}{a}=\frac{1}{\cosh \xi_{0}}$.

In terms of these, the formula for the rise velocity of the ovary ellipsoid (which is now denoted by $F r$ ) is given by (2.33) and that of the planetary ellipsoid is given by (2.34):

$$
-F r^{2} e^{2} f_{1}^{2}(e)+\frac{1}{2}\left(1-e^{2}\right)-\frac{8 F r}{R_{\mathrm{G}}} e\left[f_{2}(e)\left(\frac{1}{2}+\frac{e^{2}}{1-e^{2}}\right)+\frac{e^{2} f_{1}(e)}{1-e^{2}}\right]=\frac{8}{E_{o}} \frac{e^{2}}{1-e^{2}},
$$




\section{ARTICLE IN PRESS}

$$
-F^{2} e^{2} f_{1}^{2}(e)+\frac{1}{2} \sqrt{1-e^{2}}-\frac{8 F r}{R_{\mathrm{G}}} e\left[f_{2}(e)\left(\frac{1}{2}-e^{2}\right)-e^{2} f_{1}(e)\right]=-\frac{8}{E_{o}} e^{2} \sqrt{1-e^{2}} .
$$

In these equations, the first term in the left hand side denotes the kinetic energy due to the inertia (the pressure), the second is the gravity potential, the third is the normal viscous stress and the right hand side denotes the surface tension. The quadratic equations in $F r,(2.33)$ and (2.34), lead to the formula of the spherical bubble in the limit of $e \rightarrow 0$ ( $\xi_{0} \rightarrow \infty$ with $a$ fixed).

The aspect ratio (or shape parameter) $e$ is to be selected for a best fit to the experiment of Viana et al. There is no way that the formula (2.34) for the planetary ellipsoid can be made to fit the data; for example, the dependence on an increase of $E_{o}$ is such as to reduce the rise velocity whereas an increase, compatible with (2.33), is observed. We shall now confine our attention to the formula (2.33).

The formula (2.33) goes to the following equation in the limit $R_{\mathrm{G}} \rightarrow \infty$ :

$$
F r_{\infty}=\frac{1}{e f_{1}(e)} \sqrt{\frac{1}{2}\left(1-e^{2}\right)-\frac{8}{E_{o}} \frac{e^{2}}{1-e^{2}}} .
$$

For small $R_{\mathrm{G}}$, the formula (2.33) may be approximated by a linear equation in $F r / R_{\mathrm{G}}$ to give the solution:

$$
F r=\frac{\left(1-e^{2}\right)^{2}-16 e^{2} / E_{o}}{f_{2}(e)\left(1+e^{2}\right)+2 e^{2} f_{1}(e)} \frac{R_{\mathrm{G}}}{8 e}
$$

whence $\mathrm{Fr} \rightarrow 0$ as $R_{\mathrm{G}} \rightarrow 0$.

When $F r=0,(2.33)$ is reduced to the equation:

$$
E_{o}=16 \frac{e^{2}}{\left(1-e^{2}\right)^{2}} \text {. }
$$

If we put $E_{o}=4$ as noted in Section 1.4 , we have $e=0.41$, thus $0.41 \leqslant e<1$ for $4 \leqslant E_{o}$, which means that the bubble may be an ovary ellipsoid. It is noted here that (2.37) gives $F r_{\infty}=0$ in (2.35) and $F r=0$ in (2.36), which leads to the condition that $E_{o} \geqslant 16 e^{2} /\left(1-e^{2}\right)^{2}$ for a positive or zero solution $F r$ to the quadratic equation (2.33).

In the limit $e \rightarrow 0$ Eq. (2.33) describes the rise velocity of a perturbed spherical cap bubble. To obtain this perturbation formula we note that

$$
\left.\begin{array}{l}
\frac{c}{a}=\frac{1}{\cosh \xi_{0}}=e, \quad \frac{b}{c}=\sinh \xi_{0}=\frac{\sqrt{1-e^{2}}}{e} \\
e f_{1}(e)=\frac{1}{2} e f_{2}(e) \sim \frac{3}{2}\left(1-\frac{1}{5} e^{2}-\frac{8}{175} e^{4}+\cdots\right) .
\end{array}\right\}
$$

After inserting these expressions into (2.33) retaining terms proportional to $e^{2}$, we find that

$$
\frac{9}{4} F r^{2}+\frac{12 F r}{R_{\mathrm{G}}}-\frac{1}{2}=e^{2}\left\{\frac{8}{E_{o}}+\frac{1}{2}-\frac{9}{10} F r^{2}+\frac{168}{5} \frac{F r}{R_{\mathrm{G}}}\right\} .
$$

The leading order terms on the left were obtained by Joseph but the perturbation terms on the right are different. The curvature $s$ in Eq. (1.4) is related to the aspect ratio by

$$
s=-e^{2} / 2\left(1-e^{2}\right),
$$

whence, to leading order, we find that $e^{2}=-2 s$. 


\section{ARTICLE IN PRESS}

\section{Comparison of theory and experiment}

Viana et al. (2003) made experiments of Taylor bubbles as shown in Fig. 6. Their data maybe expressed as a functional relationship between three parameters $F r, E_{o}$ and $R_{\mathrm{G}}$ as in Eqs. (1.1)(1.3). We may plot this data for a fixed value of $E_{o}$, giving $F r$ versus $R_{\mathrm{G}}$ as is shown in $\log -\log$ scaling in Fig. 7.

In Fig. 8, we have plotted data $F r$ versus $R_{\mathrm{G}}$ for 12 values of $E_{o}$. It is important to note what was done with this data by Viana et al. (2003) and what we do with it here. Viana et al. (2003) identified a slope region for small $R_{\mathrm{G}}<10$, a flat region for $R_{\mathrm{G}}>200$ and a transition between. The flat region and the slope region give rise to power laws which were merged into the transition region using a logistic dose curve. This type of fitting was discussed briefly in the introduction to the paper and extensively by Viana et al. (2003).

Here, in Fig. 8, we plot $F r$ versus $R_{\mathrm{G}}$ data for 12 values of $E_{o}$ but we fit this data with the analytic expression (2.33) for an ovary ellipsoid rather than to power laws. The aspect ratio $e\left(E_{o}\right)$ is a fitting parameter and is listed in Table 1 , in which $e_{o}=e\left(E_{o}\right)$ is the value of $e$ in (2.33) selected as the value which most closely fit the data of Viana et al. (2003) for the 12 cases in Fig. 8. This software also gives the value $F r_{\infty}$ in (2.35) and it leads to the correlation

$$
e_{o}=E_{o}^{0.0866} / 0.357
$$

where the deviation in given by $\delta e=e-e_{o}$. The success of this procedure is impressive.

The processing of data for Table 1 is represented graphically in Fig. 9.

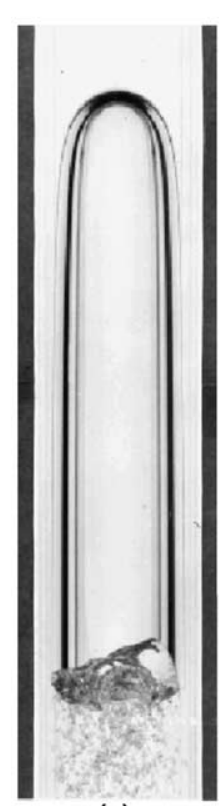

(a)

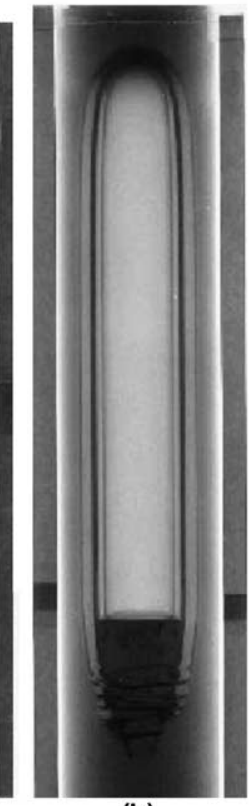

(b)

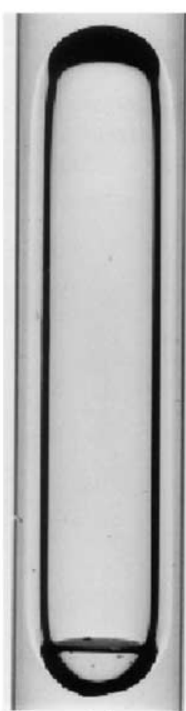

(c)

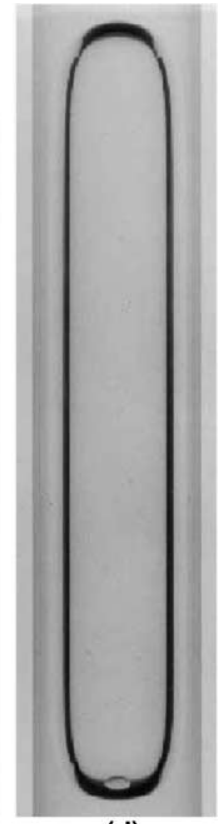

(d)

Fig. 6. Photographs of Taylor bubbles rising through $76.2 \mathrm{~mm}$ inside diameter pipe filled with different viscosity liquids. This figure is quoted from Viana et al. (2003). 


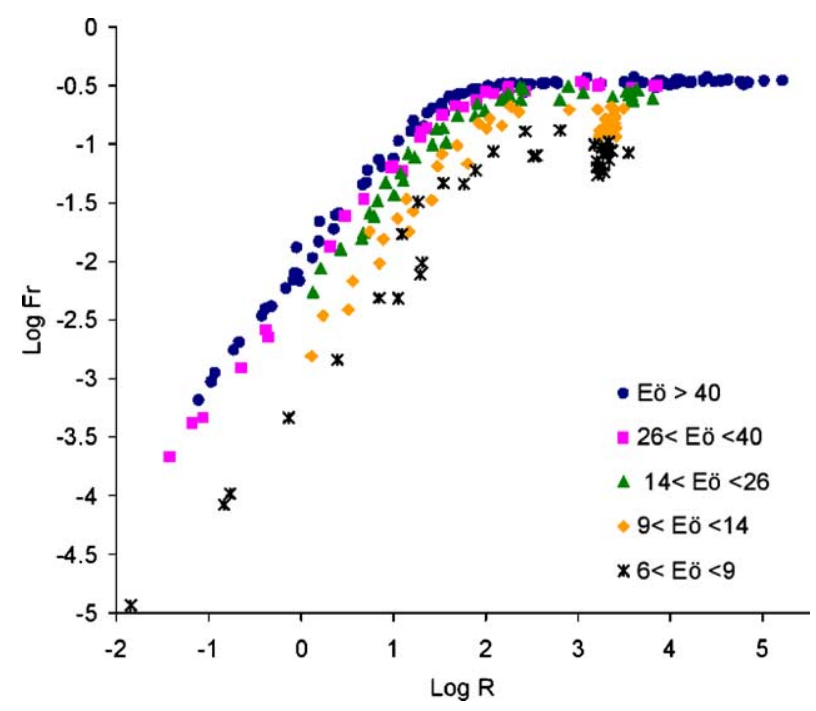

Fig. 7. $\log F r$ versus $\log R_{\mathrm{G}}$, which is quoted from Viana et al. (2003). The data used here are all sources with $E_{o} \geqslant 6$. For the data with $E_{o}<6$ refer their Fig. 9(f).

Table 1 shows that $e_{o}$ is a very weak function of $E_{o} \geqslant 15$ with data tending to a value $e_{o}=0.6$ corresponding to an ellipsoid with

$$
b \cong 0.8 a
$$

which like the true Taylor bubble has an almost spherical cap (see Fig. 10).

\section{Comparison of theory and correlations}

The processing of data on the rise velocity of Taylor bubbles in tubes of stagnant liquids by Viana et al. (2003) leads to the correlation formula (1.3) with small errors described by Fig. 1. We may then propose that the rise velocity is accurately described by (1.3); however the shape of the bubble nose is not predicted.

The theory of the rise of ovary ellipsoidal gas bubbles in viscous liquids leads to a rigorous prediction of the aspect ratio of the ovary ellipsoid which rises with exactly the same velocity $\mathrm{Fr}$ at given values of $E_{o}$ and $R_{\mathrm{G}}$ as the Taylor bubble. The value of $e$ is determined by simultaneous for $e$ of (2.33) and (1.3) for given values of $F r, R_{\mathrm{G}}$ and $E_{o}$ (Fig. 11).

A slightly simpler solution can be written out for $R_{\mathrm{G}}>200$ in which case (1.3) is replaced with the simpler formula:

$$
F r=0.34 /\left(1+3805 / E_{o}^{3.06}\right)^{0.58}
$$

which is to be solved simultaneously with (2.33) in the limit $R_{\mathrm{G}} \rightarrow \infty$ :

$$
\frac{-F^{2} e^{6}}{\left[e+\left(1-e^{2}\right) \tanh ^{-1}(e)\right]^{2}}+\frac{1}{2}\left(1-e^{2}\right)=\frac{8}{E_{o}} \frac{e^{2}}{1-e^{2}},
$$

for $e\left(E_{o}, R_{\mathrm{G}}\right)$ for given values of $E_{o}$ (Fig. 11). 


\section{ARTICLE IN PRESS}
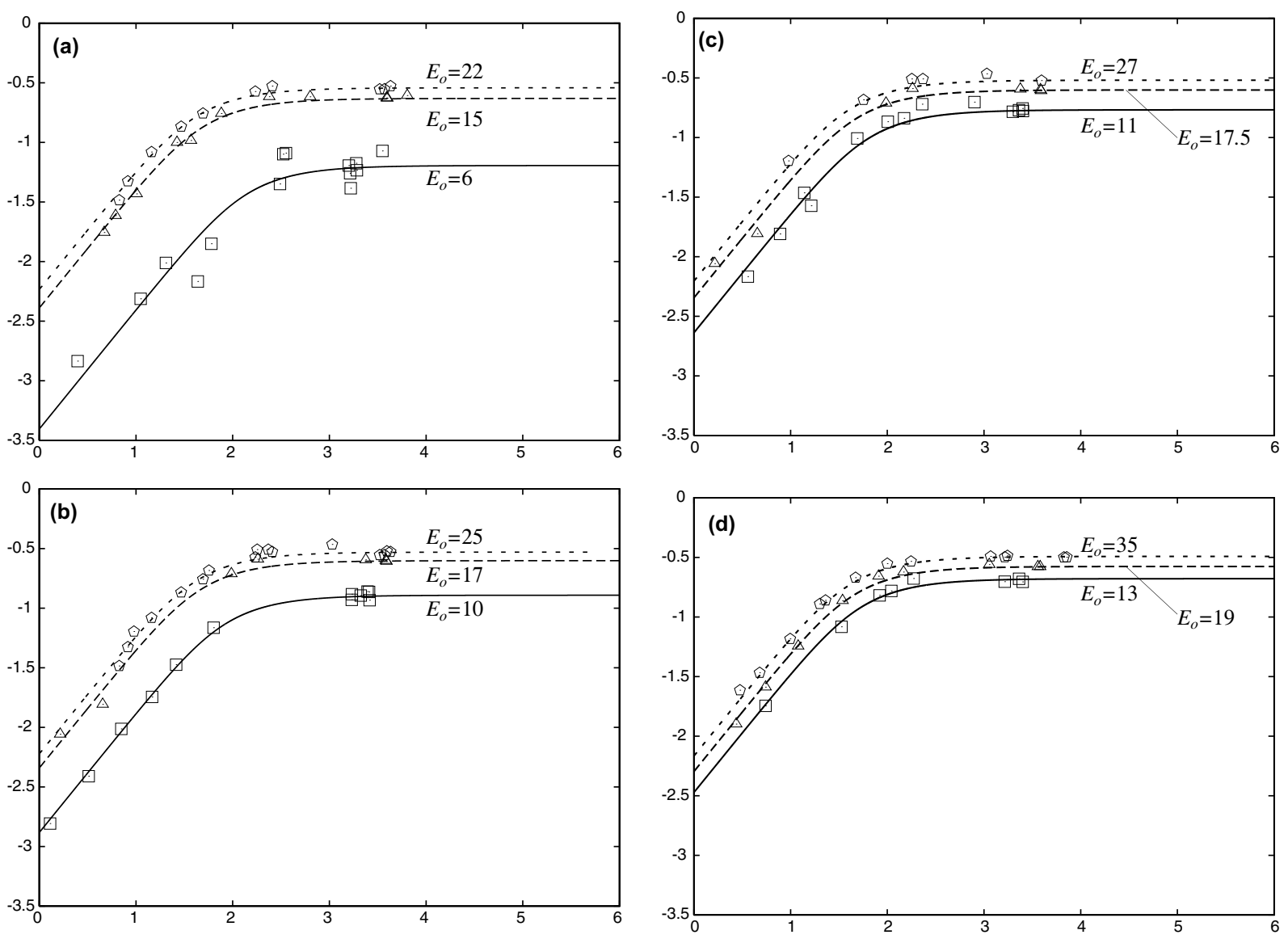

Fig. 8. $\log F r$ versus $\log R_{\mathrm{G}}$ for 12 values of values of $E_{o}$. The lines (- - ), (-- -), (-) are plots of (2.33) with $e\left(E_{o}\right)$ selected for best fit as described in Table 1 .

Table 1

Selection of $e\left(E_{o}\right)$ for 12 cases of $E_{o}$

\begin{tabular}{llllll}
\hline$E_{o}$ & $e$ & $\delta e$ & $F r_{\infty}$ & $\log F r_{\infty}$ & Fig. 8 \\
\hline 6 & 0.471243 & -0.042313 & 0.06391207 & -1.194417 & -0.891313 \\
10 & 0.536543 & -0.000228 & 0.128435 & -0.767732 & $(\mathrm{a})$ \\
11 & 0.538667 & -0.002551 & 0.170713 & -0.678617 & $(\mathrm{~b})$ \\
13 & 0.547626 & -0.001474 & 0.209595 & -0.631272 & $(\mathrm{c})$ \\
15 & 0.555870 & -0.000073 & 0.233737 & -0.601570 & $(\mathrm{a})$ \\
17 & 0.563567 & 0.001559 & 0.250282 & -0.601040 & $(\mathrm{~b})$ \\
17.5 & 0.567265 & 0.003855 & 0.250587 & -0.575845 & $(\mathrm{c})$ \\
19 & 0.568630 & 0.003855 & 0.265555 & -0.542334 & $(\mathrm{~d})$ \\
22 & 0.571912 & -0.002768 & 0.286857 & -0.529518 & $(\mathrm{a})$ \\
25 & 0.580741 & -0.000333 & 0.295449 & -0.4918728 & $(\mathrm{~b})$ \\
27 & 0.583492 & -0.001466 & 0.302880 & & (c) \\
35 & 0.593930 & -0.004315 & 0.322398 & (d) \\
\hline
\end{tabular}

The deviation is given by $\delta e=e-e_{o}$ with (3.1) and $F r_{\infty}$ is computed by (2.35). 


\section{ARTICLE IN PRESS}

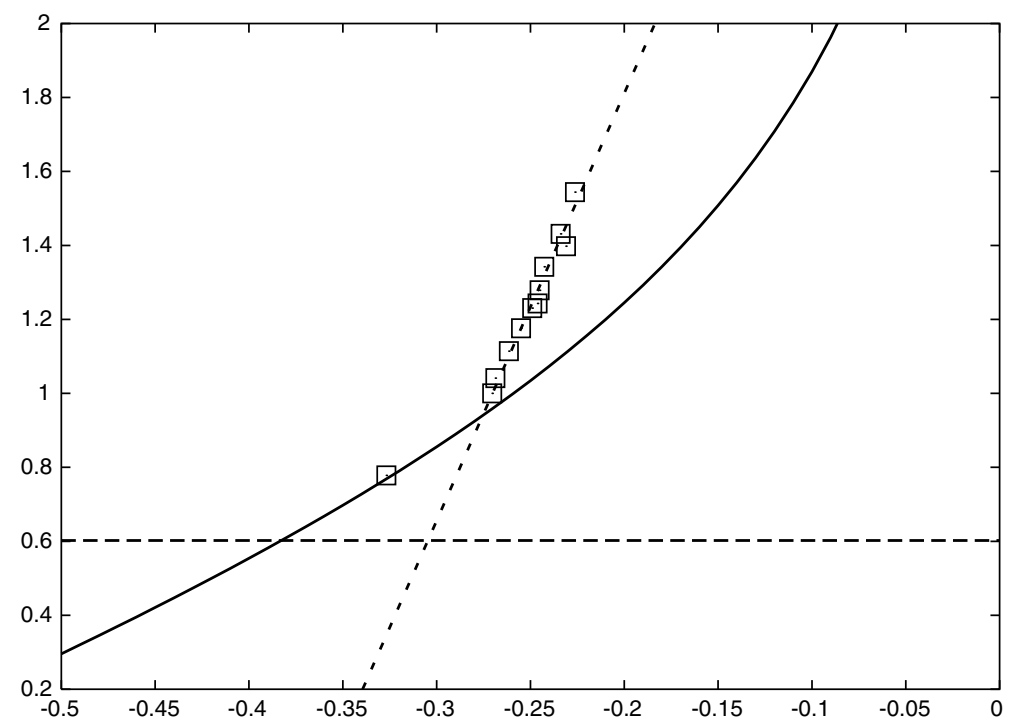

Fig. 9. $\log E_{o}$ versus $\log e$; $\square$ denotes the data given in Table 1 . The solid curve denotes the border $F r=0$ given by (2.37), above which one positive solution of $F r$ may exist and below which there arise two negative solutions or complex solutions which are meaningless. The dashed line is for $E_{o}=4$. The dotted line $\operatorname{denotes} \log e=0.0865513 \log E_{o}-$ 0.356762 for which $e_{o}=E_{o}^{0.0866} / 0.357$.

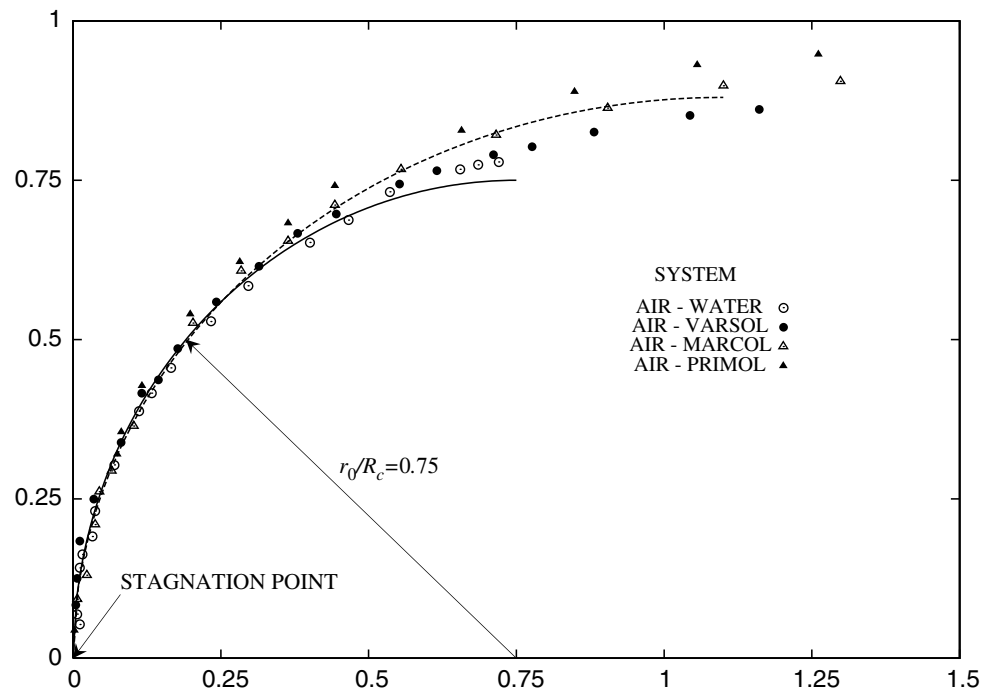

Fig. 10. Comparison of Brown's 1965 measurements of the shape of a large Taylor bubble rising in a round tube with an ovary ellipsoid (denoted by the dashed line) with $e=c / a=0.6, b=a \sqrt{1-e^{2}}=0.8 a, a=1.10$. Ovary ellipsoids with smaller $e$ are more spherical. 


\section{ARTICLE IN PRESS}

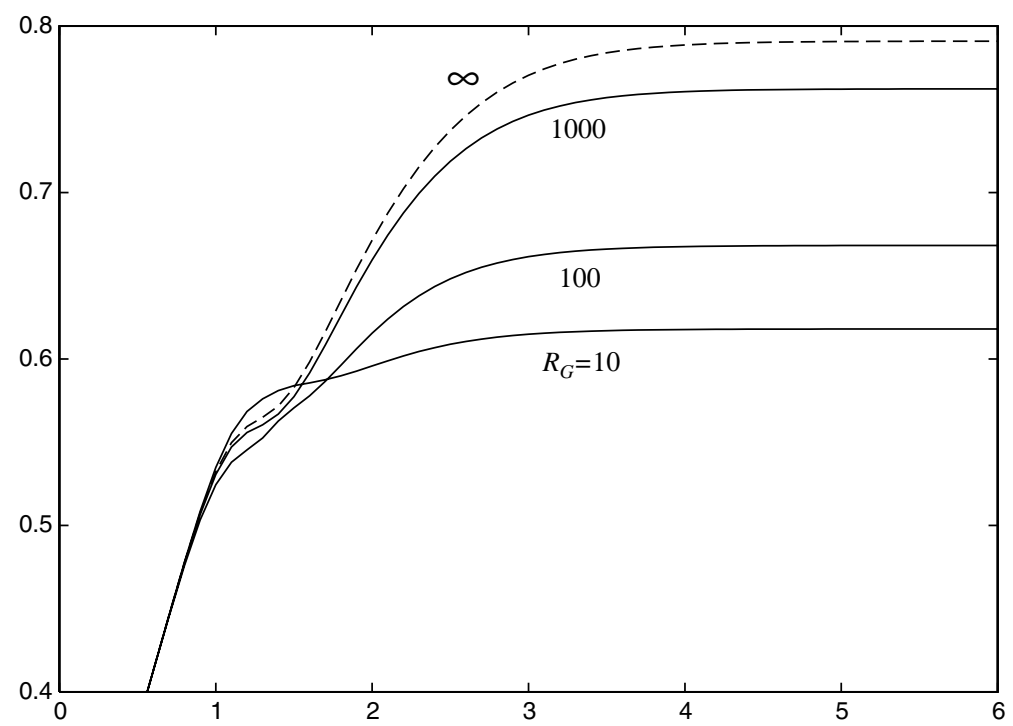

Fig. 11. $e\left(E_{o}, R_{\mathrm{G}}\right)$ versus $\log E_{o}$ for various values of $R_{\mathrm{G}}$. The curves $(-)$ are obtained by the simultaneous equations (1.3) and (2.33). The solution of (4.1) and (4.2) is shown as (- - ); all the solutions (1.3) and (2.33) coincide with (- - -) when $R_{\mathrm{G}}>10^{4}$.

\section{Conclusion}

A formula is derived giving the rise velocity of an ellipsoidal gas bubble in a viscous liquid assuming that the motion of the liquid is irrotational. The rise velocity is expressed by a Froude number and it is determined by a Reynolds number, an Eötvös number and the aspect ratio of the ellipsoid. The formula for the ovary ellipsoid was fit to the data of Viana et al. (2003) who correlated all the published data on the rise velocity of long gas bubbles in round tubes filled with viscous liquids. This data is accurately represented by our formula when the aspect ratio takes on certain values. The fitting generates a family of aspect ratios which depends strongly on the Eötvös number and less strongly on the Reynolds number; this shows that the change in the shape of the nose of the rising bubble is strongly influenced by surface tension. Our analysis completely neglects sidewall drainage induced by the rising bubble and cannot be a precise description of the dynamics. We have generated what might be called the ovary ellipsoid model of a Taylor bubble. This model is very simple and astonishingly accurate.

\section{Acknowledgement}

This work was supported in part by the NSF under grants from Chemical Transport Systems and the DOE (engineering research program of the Department of Basic Engineering Sciences). 


\section{ARTICLE IN PRESS}

\section{References}

Barr, G., 1926. The Air Bubble Viscometer. Phil. Mag. Series 7, 395.

Batchelor, G.K., 1967. An Introduction to Fluid Dynamics. Cambridge University Press, Section 6.11.

Bhaga, T., Weber, M., 1981. Bubbles in viscous liquids: shapes, wakes and velocities. J. Fluid Mech. 105, 61-85.

Bi, Q.C., Zhao, T.S., 2001. Taylor bubbles in miniaturized circular and noncircular channels. Int. J. Multiphase Flow 27, 561-570.

Bretherton, F.P., 1961. The motion of long bubbles in tubes. J. Fluid Mech. 10, 166-188.

Brown, R.A.S., 1965. The mechanics of large gas bubbles in tubes. Can. J. Chem Engng. Q2, $217-223$.

Davies, R.M., Taylor, G.I., 1950. The mechanics of large bubbles rising through liquids in tubes. Proc. R. Soc. Lond. A 200, 375-390.

Dumitrescue, D.T., 1943. Stromung and Einer Luftbluse in Senkrechten rohr. Z. Angew. Match. Mech. 23, $139-149$.

Gibson, A.H., 1913. Long air bubbles in a vertical tube. Phil. Mag. Series 6, 952.

Grace, J.R., Harrison, D., 1967. The influence of bubble shape on the rise velocities of large bubbles. Chem. Eng. Sci. 22, 1337-1347.

Hattori, S., 1935. On motion of cylindrical bubble in tube and its application to measurement of surface tension of liquid. Rep. Aeronaut. Res. Inst., Tokyo Imp. Univ., No. 115.

Joseph, D.D., 2003. Rise velocity of spherical cap bubble. J. Fluid Mech. 488, 213-223.

Milne-Thomson, L.M., 1996. Theoretical Hydrodynamics, fifth ed. Dover Publications, pp. 486-507.

Viana, F., Pardo, R., Yánez, R., Trallero, J., Joseph, D.D., 2003. Universal correlation for the rise velocity of long gas bubbles in round pipes. J. Fluid Mech. 494, 379-398.

White, E.T., Beardmore, R.H., 1962. The velocity of rise of single cylindrical air bubbles through liquids contained in vertical tubes. Chem. Engng. Sci. 17, 351-361. 